\title{
On a semilinear elliptic eigenvalue problem
}

\author{
by Mario Michele Coclite (Bari)
}

\begin{abstract}
We obtain a description of the spectrum and estimates for generalized positive solutions of $-\Delta u=\lambda(f(x)+h(u))$ in $\Omega,\left.u\right|_{\partial \Omega}=0$, where $f(x)$ and $h(u)$ satisfy minimal regularity assumptions.
\end{abstract}

Introduction. From various points of view there is still interest in the eigenvalue problem

$$
-\Delta u=\lambda(f(x)+h(u)) \quad \text { in } \Omega,\left.\quad u\right|_{\partial \Omega}=0,
$$

where $\Omega \subset \mathbb{R}^{N}, 2 \leq N$, is bounded. Following the terminology of Krasnosel'skil we define the spectrum of $(*)$ to be the set of the values $\lambda$ for which there exist positive solutions of $(*)$. Various authors have obtained a description of the spectrum of the more general problem than $(*)$, i.e.

$$
-\Delta u=\lambda f(x, u) \quad \text { in } \Omega,\left.\quad u\right|_{\partial \Omega}=0,
$$

where $f(x, u)$ satisfies some regularity hypotheses and some increasing and/or convexity conditions with respect to $u$ (see, for example, $[7 ; 11 ; 13$; $14])$. When $\lambda=1$ in $(*)$, the questions of multiplicity of solutions arise. As is well known this last problem has exhaustive answers if $f(x)=0$. When $f(x) \neq 0$ the existence of solutions is in general an open question. Nevertheless if $h(u)$ increases more slowly than $u^{p}, p<2^{*}-1=(n+2) /(n-2)$, as $u \rightarrow \infty$ some multiplicity results have been obtained utilizing recent methods of the Calculus of Variations (see, for example, $[1 ; 2 ; 6 ; 15]$ ). Recently G. Bonanno and S. A. Marano in $[3 ; 4]$ have demonstrated, together with an existence result for $(*)$, also an estimate from below of the supremum of the spectrum of $(*)$.

In this paper we obtain, under minimal assumptions on $f(x)$ and $h(u)$, a description of the spectrum and estimates of the generalized positive solu-

1991 Mathematics Subject Classification: 35J25, 35J60, 35J65.

Key words and phrases: semilinear elliptic equations, nonlinear boundary-value problems, positive solutions, supersolution and subsolution method.

Work supported by M.U.R.S.T. Italy (fondi 40\%, 60\% ) and by G.N.A.F.A. of C.N.R. 
tions of $(*)$ near $\partial \Omega$. Some results of the author (see $[8 ; 9 ; 10])$ are applied together with the method of sub-super solutions.

In the first section the main results are stated. Their proof and certain auxiliary results are contained in the second section.

1. Results. Let $\Omega \subset \mathbb{R}^{N}, 2 \leq N$, be a bounded domain with $C^{2}$ boundary. $M^{r, p}(\Omega), N<r, 2<p$, denotes the space of all $\gamma \in L_{\text {loc }}^{r}(\Omega)$ such that

$$
\varlimsup_{x \rightarrow \partial \Omega}|\gamma(x)| d(x)|\ln d(x)|^{p}<\infty, \quad d(x):=\operatorname{dist}(x, \partial \Omega) .
$$

$M^{r, p}(\Omega)$ is not empty and

$$
L^{\infty}(\Omega) \subset M^{r, p}(\Omega) \subset L^{1}(\Omega), \quad M^{r, p}(\Omega) \not \subset L^{q}(\Omega), \quad 1<q<\infty
$$

(see $[8$, Lemma 1]).

Let $|\cdot|_{p}$ be the norm of $L^{p}(\Omega), 1 \leq p<\infty$, and $|\cdot|_{\infty}$ denote the norm of $L^{\infty}(\Omega)$ and $C(\bar{\Omega})$. As usual we put $\mathbb{N} \backslash\{0\}=\mathbb{N}^{*}$ and given $\alpha, \beta \in C(\bar{\Omega})$ with $\alpha \leq \beta,[\alpha, \beta]$ denotes the set of $v \in C(\bar{\Omega})$ such that $\alpha \leq v \leq \beta$. Let $\varphi(x)$ be a positive eigenfunction of the Dirichlet problem for $-\Delta$ in $\Omega$.

The main result of this paper is the following:

Theorem. Let $f \in M^{r, p}(\Omega), f \geq 0, f \neq 0$, and $h \in C\left(\mathbb{R}_{+}\right), h \geq 0$. Define $\Lambda$ to be the set of $\lambda>0$ so that the problem

$\left(\mathrm{P}_{\lambda}\right) \quad\left\{\begin{array}{l}-\Delta u=\lambda(f(x)+h(u)), \quad u>0 \quad \text { in } \Omega ;\left.\quad u\right|_{\partial \Omega}=0, \\ u \in W_{\text {loc }}^{2, r}(\Omega) \cap C^{1}(\bar{\Omega}),\end{array}\right.$

has at least one solution. There exists $\left.\left.\lambda^{*} \in\right] 0, \infty\right]$ such that

$$
] 0, \lambda^{*}[\subset \Lambda \subset] 0, \lambda^{*}\right] \text {. }
$$

Moreover, for each solution $u$ of $\left(\mathrm{P}_{\lambda}\right)$ there exists $c=c(\lambda)>0$ such that

$$
c^{-1} \varphi \leq u \leq c \varphi \text {. }
$$

Finally,

$$
\begin{gathered}
\lim _{u \rightarrow \infty} h(u) / u=0 \Rightarrow \lambda^{*}=\infty ; \\
\varliminf_{u \rightarrow \infty} h(u) / u>0 \Rightarrow \lambda^{*}<\infty .
\end{gathered}
$$

Remark. If $f \in M^{r, p}(\Omega) \cap C^{0, \mu}(\Omega), h \in C^{0, \mu}\left(\mathbb{R}_{+}^{*}\right) \cap C\left(\mathbb{R}_{+}\right)$and $0<$ $\mu<1$ then every solution of $\left(\mathrm{P}_{\lambda}\right)$ is a classical solution, i.e. it belongs to $C^{2}(\Omega) \cap C^{1}(\bar{\Omega})$.

2. Preparatory results and proof of the Theorem. Let $G(x, y)$ be the Green function of $-\Delta$ with the Dirichlet condition on $\partial \Omega$. From the properties of $G(x, y)$ and $\varphi(x)$ it follows that there exists a continuous 
extension of $G(x, y) / \varphi(x)$ to $\bar{\Omega} \times \bar{\Omega} \backslash\left\{(x, x) \mid x \in \mathbb{R}^{N}\right\}$ (see [8; 12]), which we denote as $N(x, y)$. Let $G$ and $N$ be the operators

$$
G(v)(x)=\int_{\Omega} G(x, y) v(y) d y, \quad N(v)(x)=\int_{\Omega} N(x, y) v(y) d y .
$$

From Corollary 12 and Lemma 14 of [8] it follows that

$$
M^{r, p}(\Omega) \subset \operatorname{Dom} G, \quad M^{r, p}(\Omega) \subset \operatorname{Dom} N .
$$

Theorem 1 ([8, Lemma 13; 9, Theorems 5 and 6$])$. (1) $G(v)$ and $N(v)$ belong to $C(\bar{\Omega})$ for all $v \in M^{r, p}(\Omega)$.

(2) For every $\mathcal{F} \subset M^{r, p}(\Omega)$ and $\beta \in M^{r, p}(\Omega)$, if $|v| \leq \beta$ a.e. in $\Omega$ for all $v \in \mathcal{F}$, then $G(\mathcal{F})$ and $N(\mathcal{F})$ are relatively compact in $C(\bar{\Omega})$.

(3) Let $v_{n} \in M^{r, p}(\Omega), n \in \mathbb{N}$, and $\beta \in M^{r, p}(\Omega)$. If $v_{n} \rightarrow v$ in measure and $\left|v_{n}\right| \leq \beta$ a.e. in $\Omega$, then $v \in M^{r, p}(\Omega)$ and $G\left(v_{n}\right) \rightarrow G(v), N\left(v_{n}\right) \rightarrow$ $N(v)$ in $C(\bar{\Omega})$.

Theorem $2([8$, Theorem 16; 9 , Theorem 8$])$. For all $f \in M^{r, p}(\Omega)$, the function $u=G(f)$ belongs to $W_{\mathrm{loc}}^{2, r}(\Omega) \cap C^{1}(\bar{\Omega})$ and it is the unique solution of the problem

$$
-\Delta u=f \quad \text { in } \Omega,\left.\quad u\right|_{\partial \Omega}=0 .
$$

Theorem 3 ([8, Theorem 9; 10, Lemma 6]). Given $f \in M^{r, p}(\Omega), f \geq 0$, $f \neq 0$ there exist $m=m(f)>0$ and $M=M(f)>0$ such that the solution $u$ of (4) satisfies the estimates

$$
m \varphi(x) \leq u(x) \leq M \varphi(x), \quad x \in \bar{\Omega} .
$$

To prove the Theorem we need some general results on semilinear problems

$$
-\Delta u=k(x, u) \quad \text { in } \Omega,\left.\quad u\right|_{\partial \Omega}=0,
$$

where $k(x, u)$ is a positive Carathéodory function defined in $\Omega \times \mathbb{R}_{+}(k(\cdot, u)$ is measurable for every $u \geq 0$, and $k(x, \cdot)$ is continuous for a.e. $x \in \Omega)$.

Theorem 4. Let $\underline{u}, \bar{u} \in C(\bar{\Omega})$ and $\beta \in M^{r, p}(\Omega)$. If

$$
v \in[\varphi \underline{u}, \varphi \bar{u}] \Rightarrow|k(\cdot, v)| \leq \beta \text { a.e. in } \Omega \text { and } N(k(\cdot, v)) \in[\underline{u}, \bar{u}],
$$

then there exists a solution $u \in W_{\text {loc }}^{2, r} \cap C^{1}(\bar{\Omega}) \cap[\varphi \underline{u}, \varphi \bar{u}]$ of (5).

Proof. Since $k(\cdot, v) \in M^{r, p}(\Omega)$ and $v \in[\varphi \underline{u}, \varphi \bar{u}]$, by Theorem 2 there exists a solution $U(v) \in W_{\text {loc }}^{2, r}(\Omega) \cap C^{1}(\bar{\Omega})$ of $(5)$ and $U(v)=G(k(\cdot, v))$. The hypothesis implies that $U(v) \in[\varphi \underline{u}, \varphi \bar{u}]$. By Theorem 1 and the Schauder Theorem, $U$ has at least one fixed point. From Theorem 2, this fixed point is a solution of (5). 
$k(x, u)$ is called sublinear as $u \rightarrow \infty$ if there exists $b \in M^{r, p}(\Omega)$ with $0<b(x)$ for a.e. $x \in \Omega$ such that

$$
\lim _{u \rightarrow \infty} \frac{k(x, u)}{b(x) u}=0
$$

uniformly with respect to a.e. $x \in \Omega$. The hypotheses of the preceding theorem are satisfied if $k(x, u)$ is sublinear as $u \rightarrow \infty$. Therefore we obtain:

THEOREM 5. If $k(x, u)$ is sublinear as $u \rightarrow \infty$ and $\sup _{0 \leq t \leq s} k(\cdot, t) \in$ $M^{r, p}(\Omega)$ for all $s \geq 0$, then there exist $R>0$ and a solution $u \in W_{\mathrm{loc}}^{2, r} \cap$ $C^{1}(\bar{\Omega}) \cap[0, R \varphi]$ of $(5)$.

Proof. Since for all $v \in C(\bar{\Omega})$ with $0 \leq v$ we have

$$
k(x, v(x)) \leq \max _{0 \leq u \leq|v|_{\infty}} k(x, u),
$$

it follows that $k(\cdot, v) \in M^{r, p}(\Omega)$. Let $U(v)=G(k(\cdot, v))$, a positive solution of $(5)$.

Now we observe that

$$
\lim _{R \rightarrow 0} \frac{1}{R} N(k(\cdot, v))=0,
$$

uniformly with respect to $v$ in $[0, R \varphi]$ and $x \in \bar{\Omega}$. For $\varepsilon>0$, there exists $s_{0}>0$ such that

$$
s_{0} \leq u \Rightarrow k(x, u) \leq \varepsilon b(x) u \text { for a.e. } x \in \Omega .
$$

Then it follows that

$$
\begin{aligned}
\left.N(k(\cdot, v))(x)\right|_{0 \leq v \leq R \varphi} & =\left(\int_{v \leq s_{0}}+\int_{s_{0} \leq v}\right) N(x, y) k(y, v(y)) d y \\
& \leq\left|N\left(\sup _{0 \leq v \leq s_{0}} k(\cdot, v)\right)\right|_{\infty}+\left.\varepsilon N(b v)(x)\right|_{0 \leq v \leq R \varphi} \\
& \leq\left|N\left(\sup _{0 \leq v \leq s_{0}} k(\cdot, v)\right)\right|_{\infty}+\varepsilon R|N(b \varphi)|_{\infty} .
\end{aligned}
$$

From this (7) follows.

Let $R>0$ (independent of $x$ ) be such that

$$
0 \leq v \leq R \varphi \Rightarrow 0 \leq N(k(\cdot, v)) \leq R \Leftrightarrow 0 \leq G(k(\cdot, v)) \leq R \varphi .
$$

By virtue of the previous theorem the assertion follows.

Proof of Theorem. Firstly we observe that for all $v \in C(\bar{\Omega})$ and $\lambda>0$,

$$
\lambda(f+h(v)) \in M^{r, p}(\Omega), \quad \lambda\left(f+\sup _{0 \leq u \leq|v|_{\infty}} h(u)\right) \in M^{r, p}(\Omega) .
$$

Therefore, putting $h_{0}:=\sup \left\{\left.h(s)|0 \leq s \leq| \varphi\right|_{\infty}\right\}$, from Corollary 12 of [8] we have $\left|N\left(f+h_{0}\right)\right|_{\infty}<\infty$. 
Now the proof is divided into five steps.

STEP 1. Since for every $v \in[0, \varphi]$ we have

$$
0 \leq N[\lambda(f+h(v))](x) \leq \lambda\left|N\left(f+h_{0}\right)\right|_{\infty} \leq 1,
$$

from Theorem 4 we conclude that $\left(\mathrm{P}_{\lambda}\right)$ has at least one solution. Then

$$
] 0,1 /\left|N\left(f+h_{0}\right)\right|_{\infty}\right] \subset \Lambda .
$$

SteP 2. To prove that $\Lambda$ is an interval we show that

$$
\lambda \in \Lambda, 0<\mu<\lambda \Rightarrow \mu \in \Lambda \text {. }
$$

Let $u_{\lambda}$ be a solution of $\left(\mathrm{P}_{\lambda}\right)$, and consider the function

$$
k(x, u)=\mu\left(f(x)+h\left(\min \left\{u, u_{\lambda}(x)\right\}\right)\right) .
$$

The following properties are valid:

$$
\begin{aligned}
& 0 \leq k(x, u), \quad k(x, u) \neq 0 ; \\
& 0 \leq k(\cdot, u) \in M^{r, p}(\Omega) ; \\
& 0 \leq k(x, u) \quad \text { sublinear as } u \rightarrow \infty .
\end{aligned}
$$

From Theorem 5 we know that there exists $u_{\mu} \in W_{\text {loc }}^{2, r}(\Omega) \cap C^{1}(\bar{\Omega})$ such that

$$
-\Delta u_{\mu}=k\left(x, u_{\mu}\right), \quad 0<u_{\mu} \quad \text { in } \Omega,\left.\quad u_{\mu}\right|_{\partial \Omega}=0 .
$$

Now we prove that $u_{\mu} \leq u_{\lambda}$. Otherwise $A=\left\{x \in \Omega \mid u_{\mu}(x)>u_{\lambda}(x)\right\} \neq \emptyset$. Since

$$
\begin{aligned}
x \in A \Rightarrow-\Delta u_{\mu} & =\mu\left(f(x)+h\left(\min \left\{u_{\mu}(x), u_{\lambda}(x)\right\}\right)\right) \\
& \leq \lambda\left(f(x)+h\left(u_{\lambda}(x)\right)\right)=-\Delta u_{\lambda},
\end{aligned}
$$

we obtain

$$
-\Delta\left(u_{\mu}-u_{\lambda}\right) \leq 0 \quad \text { in } A \quad \text { and }\left.\quad\left(u_{\mu}-u_{\lambda}\right)\right|_{\partial A}=0 .
$$

By the Maximum Principle (see [5]), $u_{\mu} \leq u_{\lambda}$ in $A$. But this is not true since $A \neq \emptyset$. Therefore $u_{\mu} \leq u_{\lambda}$.

We conclude that $u_{\mu}$ is a solution of $\left(\mathrm{P}_{\lambda}\right)$, and so $\mu \in \Lambda$.

SteP 3. The estimate for positive solutions of $\left(\mathrm{P}_{\lambda}\right)$ follows by Theorem 3 .

STEP 4. Let $\lim _{u \rightarrow \infty} h(u) / u=0$; the Carathéodory function

$$
k(x, u):=\lambda(f(x)+h(u))
$$

is positive and sublinear. In fact, the function $b(x):=1+f(x)$ belongs to $M^{r, p}(\Omega)$ and (6) is satisfied. From the previous theorem, $\left(\mathrm{P}_{\lambda}\right)$ has at least one solution $u$. Moreover, if $\underline{u} \in W_{\text {loc }}^{2, r}(\Omega) \cap C^{1}(\bar{\Omega})$ is a solution of

$$
-\Delta u=f(x), \quad u>0 \quad \text { in } \Omega,\left.\quad u\right|_{\partial \Omega}=0,
$$

(see Theorem 2), from the Maximum Principle we deduce $\lambda \underline{u} \leq u$. Since by virtue of Theorem $3, \underline{u}>0$, we conclude that $u>0$. 
SteP 5. Let $\underline{\lim }_{u \rightarrow \infty} h(u) / u>0$. There exist $s_{0} \geq 0$ and $m>0$ such that $h(u) \geq m u$ for $u \geq s_{0}$. Arguing by contradiction, suppose that $\lambda^{*}=\infty$. From the Maximum Principle (see [5]) it follows that $\lambda \underline{u} \leq u_{\lambda}$. Let $\lambda_{0}>0$ be such that the open set $T=\left\{x \in \Omega \mid s_{0}<\lambda_{0} \underline{u}(x)\right\}$ is not empty. Hence, putting $\Omega_{\lambda}=\left\{x \in \Omega \mid s_{0}<u_{\lambda}(x)\right\}$, we obtain

$$
\lambda_{0} \leq \lambda \Rightarrow T \subset \Omega_{\lambda} \Rightarrow 0<|T| \leq\left|\Omega_{\lambda}\right| \text {. }
$$

Then

$$
\int_{\Omega_{\lambda}} u_{\lambda} \varphi d x \geq \lambda \int_{T} \underline{u} \varphi d x \geq \lambda \frac{s_{0}}{\lambda_{0}} \int_{T} \varphi d x
$$

and $\int_{T} \varphi d x>0$ (see [8, Theorem 9]) imply

$$
\lim _{\lambda \rightarrow \infty} \int_{\Omega_{\lambda}} u_{\lambda} \varphi d x=\infty
$$

Therefore since $u_{\lambda}$ is a solution of $\left(\mathrm{P}_{\lambda}\right)$ it follows that

$$
\lambda_{1} \int_{\Omega} u_{\lambda} \varphi d x=\lambda \int_{\Omega} f \varphi d x+\lambda \int_{\Omega} h\left(u_{\lambda}\right) \varphi d x \geq \lambda \int_{\Omega} f \varphi d x+\lambda m \int_{\Omega_{\lambda}} u_{\lambda} \varphi d x .
$$

Then

$$
\begin{aligned}
& \lambda_{1} \int_{\Omega_{\lambda}} u_{\lambda} \varphi d x+ \lambda_{1} \int_{\Omega \backslash \Omega_{\lambda}} u_{\lambda} \varphi d x \geq \lambda \int_{\Omega} f \varphi d x+\lambda m \int_{\Omega_{\lambda}} u_{\lambda} \varphi d x \\
& \Rightarrow\left(\lambda_{1}-\lambda m\right) \int_{\Omega_{\lambda}} u_{\lambda} \varphi d x+\lambda_{1} s_{0} \int_{\Omega \backslash \Omega_{\lambda}} \varphi d x \geq \lambda \int_{\Omega} f \varphi d x .
\end{aligned}
$$

This inequality is impossible, because, from (8), the first term goes to $-\infty$ as $\lambda \rightarrow \infty$. Therefore the original assumption is false. Thus $\lambda^{*}<\infty$.

\section{References}

[1] A. Ambrosetti, A perturbation theorem for superlinear boundary value problems, Math. Res. Center, Univ. of Wisconsin-Madison, Tech. Sum. Report $\sharp 1446$ (1974).

[2] A. Bahri and H. Berestycki, A perturbation method in critical point theory and applications, Trans. Amer. Math. Soc. 267 (1981), 1-32.

[3] G. Bonanno, Semilinear elliptic eigenvalue problems, preprint, 1995.

[4] G. Bonanno and S. A. Marano, Positive solutions of elliptic equations with discontinuous nonlinearities, Topol. Methods Nonlinear Anal. 8 (1996), 263-273.

[5] J. M. Bony, Principe du maximum dans les espaces de Sobolev, C. R. Acad. Sci. Paris Sér. A 265 (1967), 333-336.

[6] H. Brezis and L. Nirenberg, Positive solutions of nonlinear elliptic equations involving critical Sobolev exponents, Comm. Pure Appl. Math. 36 (1983), 437-477.

[7] K. J. Brown and H. Budin, Multiple positive solutions for a class of nonlinear boundary value problems, J. Math. Anal. Appl. 60 (1977), 329-338.

[8] M. M. Coclite, On a singular nonlinear Dirichlet problem. II, Boll. Un. Mat. Ital. B (7) 5 (1991), 955-975. 
[9] M. M. Coclite, On a singular nonlinear Dirichlet problem. III, Nonlinear Anal. 21 (1993), 547-564.

[10] —, On a singular nonlinear Dirichlet problem. IV, ibid. 23 (1994), 925-936.

[11] M. G. Crandall and P. H. Rabinowitz, Some continuation and variational methods for positive solutions of nonlinear elliptic eigenvalue problems, Arch. Rational Mech. Anal. 58 (1975), 207-218.

[12] S. Gomes, On a singular nonlinear elliptic problem, SIAM J. Math. Anal. 17 (1986), 1359-1369.

[13] J. P. Keener and H. B. Keller, Positive solutions of convex nonlinear eigenvalue problems, J. Differential Equations 16 (1974), 103-125.

[14] H. B. Keller and D. S. Cohen, Some positone problems suggested by nonlinear heat generation, J. Math. Mech. 16 (1967), 1361-1376.

[15] P. H. Rabinowitz, Multiple critical points of perturbed symmetric functionals, Trans. Amer. Math.Soc. 272 (1982), 753-769.

Dipartimento di Matematica

Università di Bari

via Orabona 4

70125 Bari, Italy

E-mail: coclite@pascal.dm.uniba.it 